\title{
Some Arithmetic Properties of Short Random Walk Integrals
}

\author{
Jonathan M. Borwein, Dirk Nuyens, Armin Straub
}

May 11, 2011

\begin{abstract}
We study the moments of the distance traveled by a walk in the plane with unit steps in random directions. While this historically interesting random walk is well understood from a modern probabilistic point of view, our own interest is in determining explicit closed forms for the moment functions and their arithmetic values at integers when only a small number of steps is taken. As a consequence of a more general evaluation, a closed form is obtained for the average distance traveled in three steps. This evaluation, as well as its proof, rely on explicit combinatorial properties, such as recurrence equations of the even moments (which are lifted to functional equations). The corresponding general combinatorial and analytic features are collected and made explicit in the case of 3 and 4 steps. Explicit hypergeometric expressions are given for the moments of a 3 -step and 4-step walk and a general conjecture for even length walks is made.
\end{abstract}

\section{Introduction, history and preliminaries}

We consider, for various values of $s$, the $n$-dimensional integral

$$
W_{n}(s):=\int_{[0,1]^{n}}\left|\sum_{k=1}^{n} e^{2 \pi \mathrm{i} x_{k}}\right|^{s} \mathrm{~d} \boldsymbol{x}
$$

which occurs in the theory of uniform random walks in the plane, where at each step a unit-step is taken in a random direction, see Figure 1. As such, the integral (1) expresses the $s$-th moment of the distance to the origin after $n$ steps. Our interest in these integrals is from the point of view of (symbolic) computation. In particular, we seek explicit closed forms of the moment functions $W_{n}(s)$ for small $n$ as well as closed form evaluations of

${ }^{*}$ CARMA, University of Newcastle, Australia. Email: jonathan.borwein@newcastle.edu.au.

${ }^{\dagger}$ K.U.Leuven, Belgium. Email: dirk.nuyens@cs.kuleuven.be; part of this work done while a research associate at the Department of Mathematics and Statistics, University of New South Wales, Australia.

${ }^{\ddagger}$ Tulane University, New Orleans, USA. Email: astraub@tulane.edu.

${ }^{\S}$ CARMA, University of Newcastle, Australia. Email: james.wan@newcastle.edu.au. 
these functions at integer arguments. Of special interest is the case $W_{n}(1)$ of the expected distance after $n$ steps.

While the general structure of the moments and densities of the random walks studied here is well-known from a modern probabilistic point of view (for instance, the characteristic function of the distance after $n$ steps is simply the Bessel function $J_{0}^{n}$ - a fact reflected in (14) and (28)), there has been little research on the question of closed forms. This is exemplified by the fact that $W_{3}(1)$ has apparently not been evaluated in the literature before (in contrast the case $W_{2}(1)=\frac{4}{\pi}$ is easy). As a consequence of a more general result we show in Section 5 that

$$
W_{3}(1)=\frac{3}{16} \frac{2^{1 / 3}}{\pi^{4}} \Gamma^{6}\left(\frac{1}{3}\right)+\frac{27}{4} \frac{2^{2 / 3}}{\pi^{4}} \Gamma^{6}\left(\frac{2}{3}\right)
$$

where $\Gamma$ is the gamma function.

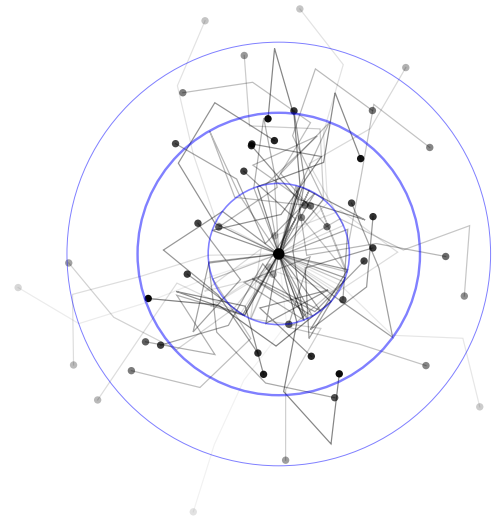

(a) Several 4-step walks

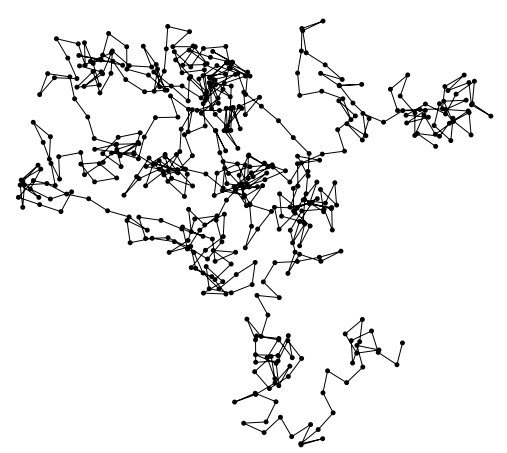

(b) A 500-step walk

Figure 1: Random walks in the plane.

A related second motivation for our work is of a numerical nature. In fact, more than 70 years after the problem was posed, [Merz79] remarks that for the densities of 4, 5 and 6-steps walks, "it has remained difficult to obtain reliable values". One challenge lies in the difficulty of computing the involved integrals, such as (28) which is highly oscillatory, to reasonably high precision. This is not straightforward, and so some comments on obtaining high precision numerical evaluations of $W_{n}(s)$ are given in Appendix A.2. A more comprehensive study of the numerics of such multiple-integrations is conducted in [BB10].

The term "random walk" first appears in a question by Karl Pearson in Nature in 1905 [Pea1905]. He asked for the probability density of a two-dimensional random walk couched in the language of how far a "rambler" (hill walker) might walk. This triggered 
a response by Lord Rayleigh [Ray1905] just one week later. Rayleigh replied that he had considered the problem earlier in the context of the composition of vibrations of random phases, and gave the probability distribution $\frac{2 x}{n} e^{-x^{2} / n}$ for large $n$. This quickly leads to a good approximation for $W_{n}(s)$ for large $n$ and fixed $s=1,2,3, \ldots$.

Another week later, Pearson again wrote in Nature, see [Pea1905b], to note that G. J. Bennett had given a solution for the probability distribution for $n=3$ which can be written in terms of the complete elliptic integral of the first kind $K$. This density function can be written as

$$
p_{3}(x)=\operatorname{Re}\left(\frac{\sqrt{x}}{\pi^{2}} K\left(\sqrt{\frac{(x+1)^{3}(3-x)}{16 x}}\right)\right),
$$

see, e.g., [Hug95] and [Pea1906]. Pearson concluded that there was still great interest in the case of small $n$ which, as he had noted, is dramatically different from that of large $n$. This is illustrated in Figure 2: while $p_{8}$ is visually almost indistinguishable from the smooth limiting form (shown in superimposed dotted lines) given by Rayleigh, the densities $p_{3}, p_{4}$ and $p_{5}$ have remarkable features of their own.

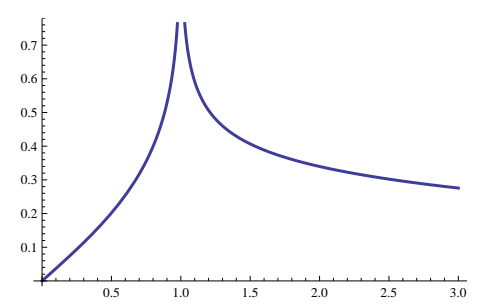

(a) $p_{3}$

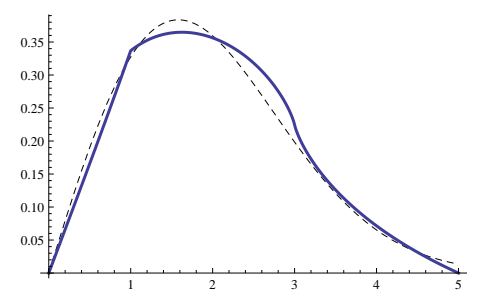

(c) $p_{5}$

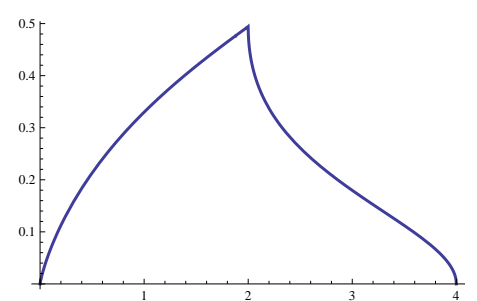

(b) $p_{4}$

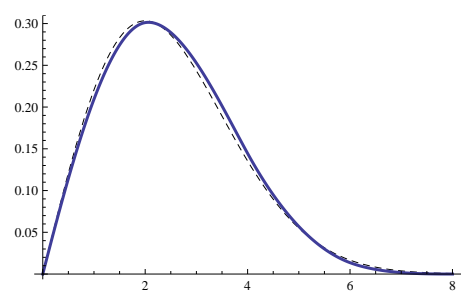

(d) $p_{8}$

Figure 2: Densities $p_{3}, p_{4}, p_{5}$ and, for contrast, $p_{8}$.

The results obtained here, as well as in a follow-up study in [BSW10], have been crucial in the discovery ([BSWZ11]) of a closed form for the density $p_{4}$ of the distance traveled in 4 steps. Additionally, an improved hypergeometric evaluation of $p_{3}$ is obtained in [BSWZ11]. 
For the convenience of the reader, the closed forms obtained in [BSWZ11] are:

$$
\begin{aligned}
& p_{3}(x)=\frac{2 \sqrt{3}}{\pi} \frac{x}{\left(3+x^{2}\right)}{ }_{2} F_{1}\left(\begin{array}{c|c}
\frac{1}{3}, \frac{2}{3} \\
1
\end{array} \mid \frac{x^{2}\left(9-x^{2}\right)^{2}}{\left(3+x^{2}\right)^{3}}\right),
\end{aligned}
$$

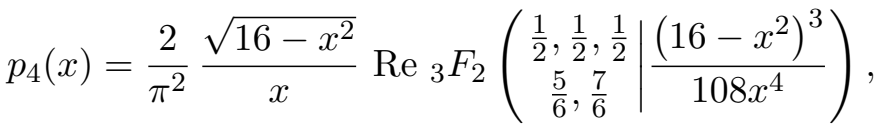

for $0 \leqslant x \leqslant 3$ and $0 \leqslant x \leqslant 4$ respectively.

It should be noted that the progress we make here (and in [BSW10, BSWZ11]) on the question of closed forms rely on techniques, for instance analysis of Meijer G-functions and their relationship with generalized hypergeometric series, that were fully developed only much later in the 20th century.

We remark that much has been done in generalizing the problem posed by Pearson. For instance, in further response to Pearson, Kluyver [Klu1906] made a lovely analysis of the cumulative distribution function of the distance traveled by a rambler in the plane for various choices of step length. Other generalizations include allowing walks in three dimensions (where the analysis is actually simpler, see [Wat41, §49]), confining the walks to different kinds of lattices, or calculating whether and when the walker would return to the origin. An excellent source of this sort of results is [Hug95].

Applications of two-dimensional random walks are numerous and well-known; for instance, [Hug95] mentions that they may be used to model the random migration of an organism possessing flagella; analysing the superposition of waves (e.g., from a laser beam bouncing off an irregular surface); and vibrations of arbitrary frequencies. The subject also finds use in Brownian motion and quantum chemistry.

We learned of the special case for $s=1$ of (1) from the whiteboard in the common room at the University of New South Wales. It had been written down by Peter Donovan as a generalization of a discrete cryptographic problem, as discussed in [Don09]. Some numerical values of $W_{n}$ evaluated at integers are shown in Tables 1 and 2. One immediately notices the apparent integrality of the sequences for the even moments - which are the moments of the squared expected distance, and where the square root for $s=2$ gives the root-meansquare distance $\sqrt{n}$. For $n=2,3,4$ these sequences were found in the Online Encyclopedia of Integer Sequences [Slo09] - the cases $n=5,6$ are in the database as a consequence of this paper.

By numerical observation, experimentation and some sketchy arguments we quickly conjectured and strongly believed that, for $k$ a nonnegative integer

$$
W_{3}(k)=\operatorname{Re}_{3} F_{2}\left(\begin{array}{c}
\frac{1}{2},-\frac{k}{2},-\frac{k}{2} \\
1,1
\end{array} \mid 4\right) .
$$

The evaluation (2) of $W_{3}(1)$ can be deduced from (6). Based on results in Sections 2 and 3, the evaluation (6) is established in Section 5. 


\begin{tabular}{r|r|r|r|r|rr}
$n$ & $s=2$ & $s=4$ & $s=6$ & $s=8$ & $s=10$ & {$[$ Slo09] } \\
\hline 2 & 2 & 6 & 20 & 70 & 252 & $\mathrm{~A} 000984$ \\
3 & 3 & 15 & 93 & 639 & 4653 & $\mathrm{~A} 002893$ \\
4 & 4 & 28 & 256 & 2716 & 31504 & $\mathrm{~A} 002895$ \\
5 & 5 & 45 & 545 & 7885 & 127905 & $\mathrm{~A} 169714$ \\
6 & 6 & 66 & 996 & 18306 & 384156 & $\mathrm{~A} 169715$
\end{tabular}

Table 1: $W_{n}(s)$ at even integers.

\begin{tabular}{c|c|c|c|c|c}
$n$ & $s=1$ & $s=3$ & $s=5$ & $s=7$ & $s=9$ \\
\hline 2 & 1.27324 & 3.39531 & 10.8650 & 37.2514 & 132.449 \\
3 & 1.57460 & 6.45168 & 36.7052 & 241.544 & 1714.62 \\
4 & 1.79909 & 10.1207 & 82.6515 & 822.273 & 9169.62 \\
5 & 2.00816 & 14.2896 & 152.316 & 2037.14 & 31393.1 \\
6 & 2.19386 & 18.9133 & 248.759 & 4186.19 & 82718.9
\end{tabular}

Table 2: $W_{n}(s)$ at odd integers.

In Section 2 we observe that the even moments $W_{n}(2 k)$ are given by integer sequences and study the combinatorial features of $f_{n}(k):=W_{n}(2 k), k$ a nonnegative integer. We show that there is a recurrence relation for the numbers $f_{n}(k)$ and confirm an observation from Table 1 that the last digit in the column for $s=10$ is always $n \bmod 10$. The discovery of (6) was precipitated by the form of $f_{3}$ given in (12).

In Section 3 some analytic results are collected, and the recursions for $f_{n}(k)$ are lifted to $W_{n}(s)$ by the use of Carlson's theorem. The recursions for $n=2,3,4,5$ are given explicitly as an example. These recursions then give further information regarding the pole structure of $W_{n}(s)$. Plots of the analytic continuation of $W_{n}(s)$ on the negative real axis are given in Figure 3. Inspired by a more general combinatorial convolution given in Section 2 we conjecture, for $n=1,2, \ldots$, the recursion

$$
W_{2 n}(s) \stackrel{?}{=} \sum_{j \geqslant 0}\left(\begin{array}{c}
s / 2 \\
j
\end{array}\right)^{2} W_{2 n-1}(s-2 j),
$$

which has been partially resolved in [BSW10].

\section{The even moments and their combinatorial features}

In the case $s=2 k$ the square root implicit in the definition (1) of $W_{n}(s)$ disappears, resulting in the fact that the even moments $W_{n}(2 k)$ are integers. In this section we collect several of the combinatorial features of these moments which, while sometimes in principle routine, provide important guidance and foundation. For instance, the combinatorial expression for $W_{3}(2 k)$ will eventually lead to the evaluation of all integer moments $W_{3}(k)$ in 


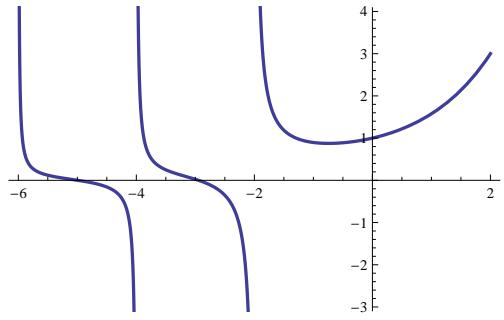

(a) $W_{3}$

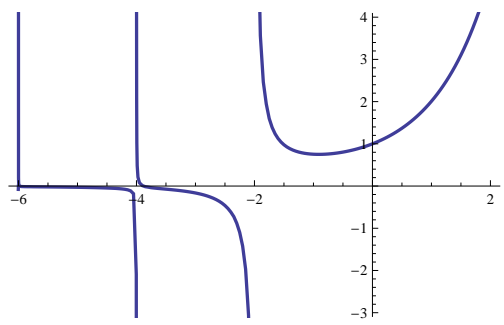

(c) $W_{5}$

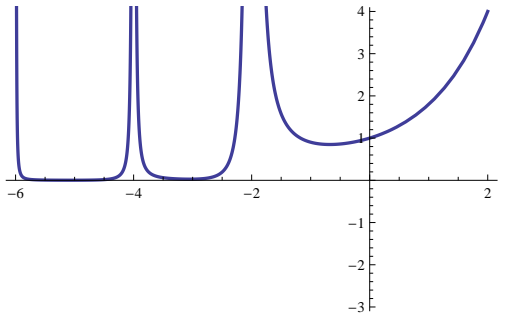

(b) $W_{4}$

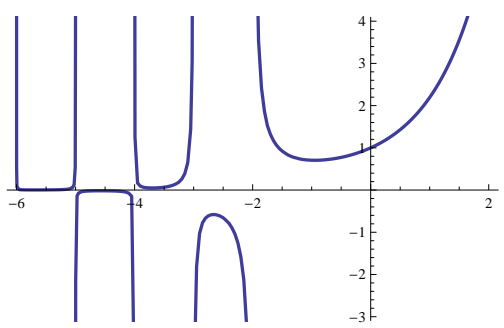

(d) $W_{6}$

Figure 3: Various $W_{n}$ and their analytic continuations.

Section 5. As a second example, the recurrence equation, in its explicit form, for $W_{4}(2 k)$ is at the heart of the derivation of the closed form (5) in [BSWZ11].

In fact, the even moments are given as sums of squares of multinomials - as is detailed next. While this result may be readily obtained from general probabilistic principles starting with the observation that the characteristic function of the distance traveled in $n$ steps is given by the Bessel function $J_{0}^{n}$ (see Section 4), we prefer to give an elementary derivation starting from the integral definition $(1)$ of $W_{n}(s)$.

Proposition 1. For nonnegative integers $k$ and $n$,

$$
W_{n}(2 k)=\sum_{a_{1}+\cdots+a_{n}=k}\left(\begin{array}{c}
k \\
a_{1}, \ldots, a_{n}
\end{array}\right)^{2} .
$$

Proof. In the spirit of the residue theorem of complex analysis, if $f\left(x_{1}, \ldots, x_{n}\right)$ has a Laurent expansion around the origin then

$$
\text { ct } f\left(x_{1}, \ldots, x_{n}\right)=\int_{[0,1]^{n}} f\left(e^{2 \pi \mathrm{i} x_{1}}, \ldots, e^{2 \pi \mathrm{i} x_{n}}\right) \mathrm{d} \boldsymbol{x},
$$

where 'ct' denotes the operator which extracts from an expression the constant term of its Laurent expansion. In light of (7), the integral definition (1) of $W_{n}(s)$ may be restated as

$$
W_{n}(s)=\operatorname{ct}\left(\left(x_{1}+\cdots+x_{n}\right)\left(1 / x_{1}+\cdots+1 / x_{n}\right)\right)^{s / 2},
$$


see also Appendix A.1. In the case $s=2 k$ the right-hand side may be finitely expanded to yield the claim: on using the multinomial theorem,

$$
\begin{aligned}
\left(x_{1}+\cdots+x_{n}\right)^{k}\left(1 / x_{1}+\cdots+1 / x_{n}\right)^{k} & \\
& =\sum_{a_{1}+\cdots+a_{n}=k}\left(\begin{array}{c}
k \\
a_{1}, \ldots, a_{n}
\end{array}\right) x_{1}^{a_{1}} \cdots x_{n}^{a_{n}} \sum_{b_{1}+\cdots+b_{n}=k}\left(\begin{array}{c}
k \\
b_{1}, \ldots, b_{n}
\end{array}\right) x_{1}^{-b_{1}} \cdots x_{n}^{-b_{n}},
\end{aligned}
$$

and the constant term is now obtained by matching $a_{1}=b_{1}, \ldots, a_{n}=b_{n}$.

Remark 1. In the case that $s$ is not an even integer, the right-hand side of (8) may still be expanded, say, when Re $s \geqslant 0$ to obtain the series evaluation

$$
W_{n}(s)=n^{s} \sum_{m \geqslant 0}(-1)^{m}\left(\begin{array}{c}
s / 2 \\
m
\end{array}\right) \sum_{k=0}^{m} \frac{(-1)^{k}}{n^{2 k}}\left(\begin{array}{c}
m \\
k
\end{array}\right) \sum_{a_{1}+\cdots+a_{n}=k}\left(\begin{array}{c}
k \\
a_{1}, \ldots, a_{n}
\end{array}\right)^{2} .
$$

An alternative elementary proof of this expansion is given in Appendix A.1. We include this alternative proof, which chronologically was our first one, because, as a side-product, it yields other interesting integral evaluations.

In light of Proposition 1, we consider the combinatorial sums

$$
f_{n}(k)=\sum_{a_{1}+\cdots+a_{n}=k}\left(\begin{array}{c}
k \\
a_{1}, \ldots, a_{n}
\end{array}\right)^{2} .
$$

of multinomial coefficients squared. These numbers also appear in [RS09] in the following way: $f_{n}(k)$ counts the number of abelian squares of length $2 k$ over an alphabet with $n$ letters (that is strings $x x^{\prime}$ of length $2 k$ from an alphabet with $n$ letters such that $x^{\prime}$ is a permutation of $x$ ). It is not hard to see that

$$
f_{n_{1}+n_{2}}(k)=\sum_{j=0}^{k}\left(\begin{array}{l}
k \\
j
\end{array}\right)^{2} f_{n_{1}}(j) f_{n_{2}}(k-j)
$$

for two non-overlapping alphabets with $n_{1}$ and $n_{2}$ letters. In particular, we may use (11) to obtain $f_{1}(k)=1, f_{2}(k)=\left(\begin{array}{c}2 k \\ k\end{array}\right)$, as well as

$$
\begin{aligned}
& f_{3}(k)=\sum_{j=0}^{k}\left(\begin{array}{c}
k \\
j
\end{array}\right)^{2}\left(\begin{array}{c}
2 j \\
j
\end{array}\right)={ }_{3} F_{2}\left(\begin{array}{c}
\frac{1}{2},-k,-k \\
1,1
\end{array} \mid 4\right)=\left(\begin{array}{c}
2 k \\
k
\end{array}\right){ }_{3} F_{2}\left(\begin{array}{c}
-k,-k,-k \\
1,-k+\frac{1}{2}
\end{array} \mid \frac{1}{4}\right), \\
& f_{4}(k)=\sum_{j=0}^{k}\left(\begin{array}{c}
k \\
j
\end{array}\right)^{2}\left(\begin{array}{c}
2 j \\
j
\end{array}\right)\left(\begin{array}{c}
2(k-j) \\
k-j
\end{array}\right)=\left(\begin{array}{c}
2 k \\
k
\end{array}\right){ }_{4} F_{3}\left(\begin{array}{c}
\frac{1}{2},-k,-k,-k \\
1,1,-k+\frac{1}{2}
\end{array} \mid 1\right) .
\end{aligned}
$$


Here and below ${ }_{p} F_{q}$ notates the generalised hypergeometric function. In general, (11) can be used to write $f_{n}$ as a sum with at most $\lceil n / 2\rceil-1$ summation indices.

We recall a generating function for $\left(f_{n}(k)\right)_{k=0}^{\infty}$ used in [BBBG08]. Let $I_{n}(z)$ denote the modified Bessel function of the first kind. Then

$$
\sum_{k \geqslant 0} f_{n}(k) \frac{z^{k}}{(k !)^{2}}=\left(\sum_{k \geqslant 0} \frac{z^{k}}{(k !)^{2}}\right)^{n}={ }_{0} F_{1}(1 ; z)^{n}=I_{0}(2 \sqrt{z})^{n} .
$$

It can be anticipated from (10) that, for fixed $n$, the sequence $f_{n}(k)$ will satisfy a linear recurrence with polynomial coefficients. A procedure for constructing these recurrences has been given in [Bar64]; in particular, that paper gives the recursions for $3 \leqslant n \leqslant 6$ explicitly. Moreover, an explicit general formula for the recurrences is given in [Ver04]:

Theorem 1. For fixed $n \geqslant 2$, the sequence $f_{n}(k)$ satisfies a recurrence of order $\lambda=\lceil n / 2\rceil$ with polynomial coefficients of degree $n-1$ :

$$
\sum_{j \geqslant 0}\left[k^{n-1} \sum_{\alpha_{1}, \ldots, \alpha_{j}} \prod_{i=1}^{j}-\alpha_{i}\left(n+1-\alpha_{i}\right)\left(\frac{k-i}{k-i+1}\right)^{\alpha_{i}-1}\right] f_{n}(k-j)=0 .
$$

Here, the sum is over all sequences $\alpha_{1}, \ldots, \alpha_{j}$ such that $0 \leqslant \alpha_{i} \leqslant n$ and $\alpha_{i+1} \leqslant \alpha_{i}-2$.

The recursions for $n=2,3,4,5$ are listed in Example 1 in Section 3.3, formulated in terms of $W_{n}(s)$ as per Theorem 4 . As a consequence of Theorem 1 we obtain:

Theorem 2. For fixed $n \geqslant 2$, the sequence $f_{n}(k)$ satisfies a recurrence of order $\lambda=\lceil n / 2\rceil$ with polynomial coefficients of degree $n-1$ :

$$
c_{n, 0}(k) f_{n}(k)+\cdots+c_{n, \lambda}(k) f_{n}(k+\lambda)=0
$$

where

$$
c_{n, 0}(k)=(-1)^{\lambda}(n ! !)^{2}\left(k+\frac{n}{4}\right)^{n+1-2 \lambda} \prod_{j=1}^{\lambda-1}(k+j)^{2},
$$

and $c_{n, \lambda}(k)=(k+\lambda)^{n-1}$. Here $n ! !=\prod_{i=0}^{\lambda-1}(n-2 i)$ is the double factorial.

Proof. The claim for $c_{n, \lambda}$ follows straight from (15). By (15), $c_{n, 0}$ is given by

$$
c_{n, 0}(k-\lambda)=\left[k^{n-1} \sum_{\alpha_{1}, \ldots, \alpha_{\lambda}} \prod_{i=1}^{\lambda}-\alpha_{i}\left(n+1-\alpha_{i}\right)\left(\frac{k-i}{k-i+1}\right)^{\alpha_{i}-1}\right]
$$

where the sum is again over all sequences $\alpha_{1}, \ldots, \alpha_{\lambda}$ such that $0 \leqslant \alpha_{i} \leqslant n$ and $\alpha_{i+1} \leqslant \alpha_{i}-2$. 
If $n$ is odd then there is only one such sequence, namely $\{n, n-2, n-4, \ldots\}$, and it follows that

$$
c_{n, 0}(k-\lambda)=(-1)^{\lambda}(n ! !)^{2} \prod_{j=1}^{\lambda-1}(k-j)^{2}
$$

in accordance with (17).

When $n=2 \lambda$ is even, there are $\lambda+1$ sequences, namely $\alpha^{0}=\{n, n-2, n-4, \ldots, 2\}$, and $\alpha^{i}$ for $1 \leqslant i \leqslant \lambda$, where $\alpha^{i}$ is constructed from $\alpha^{0}$ by subtracting all elements by 1 starting from the $(\lambda+1-i)$ th position.

Now by (18), we have

$$
c_{n, 0}(k-\lambda)=(-1)^{\lambda}\left(\prod_{i=1}^{\lambda-1}(k-i)^{2}\right) \sum_{j=0}^{\lambda}\left(\prod_{i=1}^{\lambda} a_{i}^{j}\left(n+1-a_{i}^{j}\right)\right)(k-\lambda+j),
$$

where $a_{i}^{j}$ denotes the $i$ th element of $a^{j}$.

We make the key observation that the sum in (20) is symmetric, so writing it backwards and adding that to itself, we factor out the term involving $k$ :

$$
2 \sum_{j=0}^{\lambda}\left(\prod_{i=1}^{\lambda} a_{i}^{j}\left(n+1-a_{i}^{j}\right)\right)(k-\lambda+j)=(2 k-\lambda) \sum_{j=0}^{\lambda} \prod_{i=1}^{\lambda} a_{i}^{j}\left(n+1-a_{i}^{j}\right) .
$$

As we know the sequences $a^{j}$ explicitly, the product on the right of (21) simplifies to

$$
(2 \lambda) ! \frac{\left(\begin{array}{c}
2 j \\
j
\end{array}\right)\left(\begin{array}{c}
2 \lambda-2 j \\
\lambda-j
\end{array}\right)}{\left(\begin{array}{c}
2 \lambda \\
\lambda
\end{array}\right)}
$$

Hence the sum on the right of (21) is

$$
\sum_{j=0}^{\lambda}(2 \lambda) ! \frac{\left(\begin{array}{c}
2 j \\
j
\end{array}\right)\left(\begin{array}{c}
2 \lambda-2 j \\
\lambda-j
\end{array}\right)}{\left(\begin{array}{c}
2 \lambda \\
\lambda
\end{array}\right)}=2^{2 \lambda} \lambda !^{2}
$$

which can be verified, for instance, using the snake oil method ([Wil93]). Substituting this into (20) gives (17) for even $n$.

Remark 2. For fixed $k$, the map $n \mapsto f_{n}(k)$ can be given by the evaluation of a polynomial in $n$ of degree $k$. This follows from

$$
f_{n}(k)=\sum_{j=0}^{k}\left(\begin{array}{l}
n \\
j
\end{array}\right) \sum_{\substack{a_{1}+\cdots+a_{j}=k \\
a_{i}>0}}\left(\begin{array}{c}
k \\
a_{1}, \ldots, a_{j}
\end{array}\right)^{2}
$$


because the right-hand side is a linear combination (with positive coefficients only depending on $k$ ) of the polynomials $\left(\begin{array}{c}n \\ j\end{array}\right)=\frac{n(n-1) \cdots(n-j+1)}{j !}$ in $n$ of degree $j$ for $j=0,1, \ldots, k$.

From (23) the coefficient of $\left(\begin{array}{l}n \\ k\end{array}\right)$ is seen to be $(k !)^{2}$. We therefore formally obtain the first-order approximation $W_{n}(s) \approx_{n} n^{s / 2} \Gamma(s / 2+1)$ for $n$ going to infinity, see also [Klu1906]. In particular, $W_{n}(1) \approx_{n} \sqrt{n \pi} / 2$. Similarly, the coefficient of $\left(\begin{array}{c}n \\ k-1\end{array}\right)$ is $\frac{k-1}{4}(k !)^{2}$ which gives rise to the second-order approximation

$$
(k !)^{2}\left(\begin{array}{l}
n \\
k
\end{array}\right)+\frac{k-1}{4}(k !)^{2}\left(\begin{array}{c}
n \\
k-1
\end{array}\right)=k ! n^{k}-\frac{k(k-1)}{4} k ! n^{k-1}+O\left(n^{k-2}\right)
$$

of $f_{n}(k)$. We therefore obtain

$$
W_{n}(s) \approx_{n} n^{s / 2-1}\left\{\left(n-\frac{1}{2}\right) \Gamma\left(\frac{s}{2}+1\right)+\Gamma\left(\frac{s}{2}+2\right)-\frac{1}{4} \Gamma\left(\frac{s}{2}+3\right)\right\}
$$

which is exact for $s=0,2,4$. In particular, $W_{n}(1) \approx_{n} \sqrt{n \pi} / 2+\sqrt{\pi / n} / 32$. More general approximations are given in [Cra09].

Remark 3. It follows straight from (10) that, for primes $p, f_{n}(p) \equiv n$ modulo $p$. Further, for $k \geqslant 1, f_{n}(k) \equiv n$ modulo 2 . This may be derived inductively from the recurrence (11) since, assuming that $f_{n}(k) \equiv n$ modulo 2 for some $n$ and all $k \geqslant 1$,

$$
f_{n+1}(k)=\sum_{j=0}^{k}\left(\begin{array}{l}
k \\
j
\end{array}\right)^{2} f_{n}(j) \equiv 1+\sum_{j=1}^{k}\left(\begin{array}{l}
k \\
j
\end{array}\right) n=1+n\left(2^{k}-1\right) \equiv n+1 \quad(\bmod 2) .
$$

Hence for odd primes $p$,

$$
f_{n}(p) \equiv n \quad(\bmod 2 p)
$$

The congruence $(24)$ also holds for $p=2$ since $f_{n}(2)=(2 n-1) n$, compare (23). In particular, (24) confirms that indeed the last digit in the column for $s=10$ is always $n$ mod 10 - an observation from Table 1.

Remark 4. The integers $f_{3}(k)$ (respectively $f_{4}(k)$ ) also arise in physics, see for instance [BBBG08], and are referred to as hexagonal (respectively diamond) lattice integers. The corresponding entries in Sloane's online encyclopedia [Slo09] are A002893 and A002895. We recall the following formulae [BBBG08, (186)-(188)], relating these sequences in non- 
obvious ways:

$$
\begin{aligned}
\left(\sum_{k \geqslant 0} f_{3}(k)(-x)^{k}\right)^{2} & =\sum_{k \geqslant 0} f_{2}(k)^{3} \frac{x^{3 k}}{\left((1+x)^{3}(1+9 x)\right)^{k+\frac{1}{2}}} \\
& =\sum_{k \geqslant 0} f_{2}(k) f_{3}(k) \frac{(-x(1+x)(1+9 x))^{k}}{((1-3 x)(1+3 x))^{2 k+1}} \\
& =\sum_{k \geqslant 0} f_{4}(k) \frac{x^{k}}{((1+x)(1+9 x))^{k+1}}
\end{aligned}
$$

It would be instructive to similarly engage $f_{5}(k)$ for which we have

$$
f_{5}(k)=\left(\begin{array}{c}
2 k \\
k
\end{array}\right) \sum_{j=0}^{k} \frac{\left(\begin{array}{c}
k \\
j
\end{array}\right)^{4}}{\left(\begin{array}{c}
2 k \\
2 j
\end{array}\right)} 3 F_{2}\left(\begin{array}{c}
-j,-j,-j \\
1, \frac{1}{2}-j
\end{array} \mid \frac{1}{4}\right),
$$

as follows from (11).

\section{Analytic features of the moments}

This section collects analytic features of the moments $W_{n}(s)$ as a function in $s$. In particular, it is shown that the recurrences for the even moments $W_{n}(2 k)$, described in Section 2, extend to functional equations. This is deduced in the usual way from Carlson's theorem. Still we find it instructive to give the details, especially as the explicit form of the functional equations and the resulting pole structures were crucial for discovery and proof of the closed forms in the cases $n=3,4,5$ obtained in here and in [BSW10, BSWZ11], as was true for the results in Section 2.

\subsection{Analyticity}

We start with a preliminary investigation of the analyticity of $W_{n}(s)$ for a given $n$. This analyticity also follows from the general principle that the moment functions of bounded random variables are always analytic in a strip of the complex plane containing the right half-plane - but again we prefer to give a short direct proof.

Proposition 2. $W_{n}(s)$ is analytic at least for $\operatorname{Re} s>0$.

Proof. Let $s_{0}$ be a real number such that the integral in (1) converges for $s=s_{0}$. Then we claim that $W_{n}(s)$ is analytic in $s$ for $\operatorname{Re} s>s_{0}$. To this end, let $s$ be such that $s_{0}<\operatorname{Re} s \leqslant s_{0}+\lambda$ for some real $\lambda>0$. For any real $0 \leqslant a \leqslant n$,

$$
\left|a^{s}\right|=a^{\operatorname{Re} s} \leqslant n^{\lambda} a^{s_{0}},
$$


and therefore

$$
\left.\sup _{s_{0}<\operatorname{Re} s \leqslant s_{0}+\lambda} \int_{[0,1]^{n}}|| \sum_{k=1}^{n} e^{2 \pi \mathrm{i} x_{k}}\right|^{s} \mid \mathrm{d} \boldsymbol{x} \leqslant n^{\lambda} W_{n}\left(s_{0}\right)<\infty .
$$

This local boundedness implies, see for instance [Mat01], that $W_{n}(s)$ as defined by the integral in (1) is analytic in $s$ for Re $s>s_{0}$. Since the integral clearly converges for $s=0$, the claim follows.

This result will be extended in Theorem 5 and Corollary 1.

\section{$3.2 n=1$ and $n=2$}

It follows straight from the integral definition (1) that $W_{1}(s)=1$. In the case $n=2$, direct integration of (40) with $n=2$ yields

$$
W_{2}(s)=2^{s+1} \int_{0}^{1 / 2} \cos (\pi t)^{s} \mathrm{~d} t=\left(\begin{array}{c}
s \\
s / 2
\end{array}\right),
$$

which may also be obtained using (9). In particular, $W_{2}(1)=4 / \pi$. It may be worth noting that neither Maple 14 nor Mathematica 7 can evaluate $W_{2}(1)$ if it is entered naively in form of the defining (1) (or expanded as the square root of a sum of squares), each returning the symbolic answer ' 0 '.

\subsection{Functional equations}

We may lift the recursive structure of $f_{n}$, defined in Section 2, to $W_{n}$ to a fair degree on appealing to Carlson's theorem [Tit39, 5.81]. We recall that a function $f$ is of exponential type in a region if $|f(z)| \leqslant M e^{c|z|}$ for some constants $M$ and $c$.

Theorem 3 (Carlson). Let $f$ be analytic in the right half-plane $\operatorname{Re} z \geqslant 0$ and of exponential type with the additional requirement that

$$
|f(z)| \leqslant M e^{d|z|}
$$

for some $d<\pi$ on the imaginary axis $\operatorname{Re} z=0$. If $f(k)=0$ for $k=0,1,2, \ldots$ then $f(z)=0$ identically.

Theorem 4. Given that $f_{n}(k)$ satisfies a recurrence

$$
c_{n, 0}(k) f_{n}(k)+\cdots+c_{n, \lambda}(k) f_{n}(k+\lambda)=0
$$

with polynomial coefficients $c_{n, j}(k)$ (see Theorem 2) then $W_{n}(s)$ satisfies the corresponding functional equation

$$
c_{n, 0}(s / 2) W_{n}(s)+\cdots+c_{n, \lambda}(s / 2) W_{n}(s+2 \lambda)=0,
$$

for $\operatorname{Re} s \geqslant 0$. 
Proof. Let

$$
U_{n}(s):=c_{n, 0}(s) W_{n}(2 s)+\cdots+c_{n, \lambda}(s) W_{n}(2 s+2 \lambda) .
$$

Since $f_{n}(k)=W_{n}(2 k)$ by Proposition $1, U_{n}(s)$ vanishes at the nonnegative integers by assumption. Consequently, $U_{n}(s)$ is zero throughout the right half-plane and we are doneonce we confirm that Theorem 3 applies. By Proposition 2, $W_{n}(s)$ is analytic for Re $s \geqslant 0$. Clearly, $\left|W_{n}(s)\right| \leqslant n^{\operatorname{Re} s}$. Thus

$$
\left|U_{n}(s)\right| \leqslant\left(\left|c_{n, 0}(s)\right|+\left|c_{n, 1}(s)\right| n^{2}+\cdots+\left|c_{n, \lambda}(s)\right| n^{2 \lambda}\right) n^{2 \operatorname{Re} s} .
$$

In particular, $U_{n}(s)$ is of exponential type. Further, $U_{n}(s)$ is polynomially bounded on the imaginary axis Re $s=0$. Thus $U_{n}$ satisfies the growth conditions of Theorem 3 .

Example 1. For $n=2,3,4,5$ we find

$$
\begin{aligned}
(s+2) W_{2}(s+2)-4(s+1) W_{2}(s) & =0, \\
(s+4)^{2} W_{3}(s+4)-2\left(5 s^{2}+30 s+46\right) W_{3}(s+2)+9(s+2)^{2} W_{3}(s) & =0, \\
(s+4)^{3} W_{4}(s+4)-4(s+3)\left(5 s^{2}+30 s+48\right) W_{4}(s+2)+64(s+2)^{3} W_{4}(s) & =0,
\end{aligned}
$$

and

$$
\begin{aligned}
& (s+6)^{4} W_{5}(s+6)-\left(35(s+5)^{4}+42(s+5)^{2}+3\right) W_{5}(s+4)+ \\
& \quad(s+4)^{2}\left(259(s+4)^{2}+104\right) W_{5}(s+2)-225(s+4)^{2}(s+2)^{2} W_{5}(s)=0 .
\end{aligned}
$$

We note that in each case the recursion lets us determine significant information about the nature and position of any poles of $W_{n}(s)$. We exploit this in the next theorem for $n \geqslant 3$. The case $n=2$ is transparent since as determined above $W_{2}(s)=\left(\begin{array}{c}s \\ s / 2\end{array}\right)$ which has simple poles at the negative odd integers.

Theorem 5. Let an integer $n \geqslant 3$ be given. The recursion guaranteed by Theorem 4 provides an analytic continuation of $W_{n}(s)$ to all of the complex plane with poles of at most order two at certain negative integers.

Proof. Proposition 2 proves analyticity in the right halfplane. It is clear that the recursion given by Theorem 4 ensures an analytic continuation with poles only possible at negative integer values compatible with the recursion. Indeed, with $\lambda=\lceil n / 2\rceil$ we have

$$
W_{n}(s)=-\frac{c_{n, 1}(s / 2) W_{n}(s+2)+\cdots+c_{n, \lambda}(s / 2) W_{n}(s+2 \lambda)}{c_{n, 0}(s / 2)}
$$

with the $c_{n, j}$ as in (16). We observe that the right side of (26) only involves $W_{n}(s+2 k)$ for $k \geqslant 1$. Therefore the least negative pole can only occur at a zero of $c_{n, 0}(s / 2)$ which is explicitly given in (17). We then note that the recursion forces poles to be simple or of order two, and to be replicated as claimed. 
Corollary 1. If $n \geqslant 3$ then $W_{n}(s)$, as given by (1), is analytic for $\operatorname{Re} s>-2$.

Proof. This follows directly from Theorem 5, the fact that $c_{n, 0}(s / 2)$ given in (17) has no zero for $s=-1$, and the proof of Proposition 2.

In Figure 3, on page 6, the analytic continuations for each of $W_{3}, W_{4}, W_{5}$, and $W_{6}$ are plotted on the real line.

Example 2. Using the recurrence given in Example 1 we find that $W_{3}(s)$ has simple poles at $s=-2,-4,-6, \ldots$, compare Figure $3(\mathrm{a})$. Moreover, the residue at $s=-2$ is given by $\operatorname{Res}_{-2}\left(W_{3}\right)=2 /(\sqrt{3} \pi)$, and all other residues of $W_{3}$ are rational multiples thereof. This may be obtained from the integral representation given in (29) observing that, at $s$ a negative even integer, the residue contributions are entirely from the first term.

Example 3. Similarly, we find that $W_{4}$ has double poles at $-2,-4,-6, \ldots$, compare Figure 3(b). With more work, or using a more sophisticated analysis as in [BSWZ11], it is possible to show that

$$
\lim _{s \rightarrow-2}(s+2)^{2} W_{4}(s)=\frac{3}{2 \pi^{2}},
$$

and in similar fashion the complete structure of $W_{4}(s)$ is thus accessible.

Remark 5. More generally, it would appear that Theorem 5 can be extended to show that

- for $n$ odd $W_{n}$ has simple poles at $-2 p$ for $p=1,2,3, \ldots$, while

- for $n$ even $W_{n}$ has simple poles at $-2 p$ and $2(1-p)-n / 2$ for $p=1,2,3, \ldots$ which will overlap when $4 \mid n$.

This conjecture is further investigated in [BSW10].

We close this section by remarking that the knowledge about the poles of $W_{n}$ for instance reveals the asymptotic behaviour of the densities $p_{n}$ at 0 . This is detailed in [BSWZ11] where closed forms for the densities are investigated, with particular emphasis on $n=3,4,5$. It is worth noting that $p_{5}$ was first proven rigorously not to be linear on $[0,1]$ in $[$ Fet63].

\subsection{Convolution series}

Our attempt to lift the convolution sum (11) to $W_{n}(s)$ resulted in the following conjecture:

Conjecture 1. For positive integers $n$ and complex $s$,

$$
W_{2 n}(s) \stackrel{?}{=} \sum_{j \geqslant 0}\left(\begin{array}{c}
s / 2 \\
j
\end{array}\right)^{2} W_{2 n-1}(s-2 j) .
$$


It is understood that the right-hand side of (27) refers to the analytic continuation of $W_{n}$ as guaranteed by Theorem 5 . Conjecture 1, which is consistent with the pole structure described in Remark 5, has been confirmed by David Broadhurst [Bro09] using a Bessel integral representation for $W_{n}$, given in (28), for $n=2,3,4,5$ and odd integers $s<50$ to a precision of 50 digits. By (11) the conjecture clearly holds for $s$ an even positive integer. For $n=1$ it is confirmed next.

Example 4. For $n=1$ we obtain from (27) using $W_{1}(s)=1$,

$$
W_{2}(s)=\sum_{j \geqslant 0}\left(\begin{array}{c}
s / 2 \\
j
\end{array}\right)^{2}=\left(\begin{array}{c}
s \\
s / 2
\end{array}\right)
$$

which agrees with (25).

We remark that a partial resolution of Conjecture 1 is obtained in [BSWZ11].

\section{Bessel integral representations}

As noted in the introduction, Kluyver [Klu1906] made a lovely analysis of the cumulative distribution function of the distance traveled by a "rambler" in the plane for various fixed step lengths. In particular, for our uniform walk Kluyver provides the Bessel function representation

$$
P_{n}(t)=t \int_{0}^{\infty} J_{1}(x t) J_{0}^{n}(x) \mathrm{d} x .
$$

Thus, $W_{n}(s)=\int_{0}^{n} t^{s} p_{n}(t) \mathrm{d} t$, where $p_{n}=P_{n}^{\prime}$. From here, Broadhurst [Bro09] obtains

$$
W_{n}(s)=2^{s+1-k} \frac{\Gamma\left(1+\frac{s}{2}\right)}{\Gamma\left(k-\frac{s}{2}\right)} \int_{0}^{\infty} x^{2 k-s-1}\left(-\frac{1}{x} \frac{\mathrm{d}}{\mathrm{d} x}\right)^{k} J_{0}^{n}(x) \mathrm{d} x
$$

for real $s$ and is valid as long as $2 k>s>\max \left(-2,-\frac{n}{2}\right)$.

Remark 6. For $n=3,4$, symbolic integration in Mathematica of (28) leads to interesting analytic continuations [Cra09] such as

$$
W_{3}(s)=\frac{1}{2^{2 s+1}} \tan \left(\frac{\pi s}{2}\right)\left(\begin{array}{c}
s \\
\frac{s-1}{2}
\end{array}\right)_{3}^{2} F_{2}\left(\begin{array}{c}
\frac{1}{2}, \frac{1}{2}, \frac{1}{2} \\
\frac{s+3}{2}, \frac{s+3}{2} \mid \frac{1}{4}
\end{array}\right)+\left(\begin{array}{c}
s \\
\frac{s}{2}
\end{array}\right){ }_{3} F_{2}\left(\begin{array}{c}
-\frac{s}{2},-\frac{s}{2},-\frac{s}{2} \\
1,-\frac{s-1}{2}
\end{array} \mid \frac{1}{4}\right),
$$

and

$$
W_{4}(s)=\frac{1}{2^{2 s}} \tan \left(\frac{\pi s}{2}\right)\left(\begin{array}{c}
s \\
\frac{s-1}{2}
\end{array}\right)_{4}^{3} F_{3}\left(\begin{array}{c}
\frac{1}{2}, \frac{1}{2}, \frac{1}{2}, \frac{s}{2}+1 \\
\frac{s+3}{2}, \frac{s+3}{2}, \frac{s+3}{2} \mid 1
\end{array}\right)+\left(\begin{array}{c}
s \\
\frac{s}{2}
\end{array}\right){ }_{4} F_{3}\left(\begin{array}{c}
\frac{1}{2},-\frac{s}{2},-\frac{s}{2},-\frac{s}{2} \\
1,1,-\frac{s-1}{2}
\end{array} \mid 1\right) .
$$


We note that for $s=2 k=0,2,4, \ldots$ the first term in (29) (resp. (30)) is zero and the second is a formula given in (12) (resp. (13)). Thence, one can in principle prove (29) and (30) by applying Carlson's theorem-after showing the singularities at $1,3,5, \ldots$ are removable. A rigorous proof, along with extensions and more details, appears in [BSW10].

\section{The odd moments of a three-step walk}

In this section, we combine the results of the previous sections to finally prove the hypergeometric evaluation (6) of the moments $W_{3}(k)$ in Theorem 6.

It is elementary to express the distance $y$ of an $(n+1)$-step walk conditioned on a given distance $x$ of an $n$-step walk. By a simple application of the cosine rule we find

$$
y^{2}=x^{2}+1+2 x \cos (\theta)
$$

where $\theta$ is the outside angle of the triangle with sides of lengths $x, 1$, and $y$ :

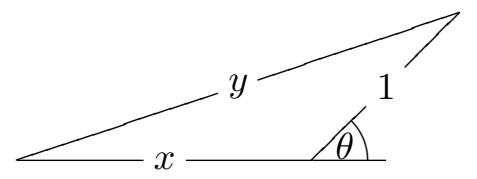

It follows that the $s$-th moment of an $(n+1)$-step walk conditioned on a given distance $x$ of an $n$-step walk is

$$
g_{s}(x):=\frac{1}{\pi} \int_{0}^{\pi} y^{s} \mathrm{~d} \theta=|x-1|_{2}^{s} F_{1}\left(\begin{array}{c}
\frac{1}{2},-\frac{s}{2} \\
1
\end{array} \mid-\frac{4 x}{(x-1)^{2}}\right) .
$$

Here we appealed to symmetry to restrict the angle to $\theta \in[0, \pi)$. We then evaluated the integral in hypergeometric form which, for instance, can be done with the help of Mathematica. Observe that $g_{s}(x)$ does not depend on $n$. Since $W_{n+1}(s)$ is the $s$-th moment of the distance of an $(n+1)$-step walk, we obtain

$$
W_{n+1}(s)=\int_{0}^{n} g_{s}(x) p_{n}(x) \mathrm{d} x
$$

where $p_{n}(x)$ is the density of the distance $x$ for an $n$-step walk. Clearly, for the 1-step walk we have $p_{1}(x)=\delta_{1}(x)$, a Dirac delta function at $x=1$. It is also easily shown that the probability density for a 2 -step walk is given by $p_{2}(x)=2\left(\pi \sqrt{4-x^{2}}\right)^{-1}$ for $0 \leqslant x \leqslant 2$ and 0 otherwise. The density $p_{3}(x)$ is given in (3).

For $n=3$, based on (12) we define

$$
V_{3}(s):={ }_{3} F_{2}\left(\begin{array}{c}
\frac{1}{2},-\frac{s}{2},-\frac{s}{2} \mid 4 \\
1,1
\end{array}\right),
$$


so that by Proposition 1 and $(12), W_{3}(2 k)=V_{3}(2 k)$ for nonnegative integers $k$. This led us to explore $V_{3}(s)$ more generally numerically and so to conjecture and eventually prove the following:

Theorem 6. For nonnegative even integers and all odd integers $k$ :

$$
W_{3}(k)=\operatorname{Re} V_{3}(k) .
$$

Remark 7. Note that, for all complex $s$, the function $V_{3}(s)$ also satisfies the recursion given in Example 1 for $W_{3}(s)$-as is routine to prove symbolically using for instance creative telescoping [PWZ06]. However, $V_{3}$ does not satisfy the growth conditions of Carlson's Theorem (Theorem 3). Thus, it yields a rather nice illustration that the hypotheses can fail.

Proof of Theorem 6. It remains to prove the result for odd integers. Since, as noted in Remark 7, for all complex $s$, the function $V_{3}(s)$ defined in (33) also satisfies the recursion given in Example 1, it suffices to show that the values given for $s=1$ and $s=-1$ are correct. From (32), we have the following expression for $W_{3}$ :

$$
W_{3}(s)=\frac{2}{\pi} \int_{0}^{2} \frac{g_{s}(x)}{\sqrt{4-x^{2}}} \mathrm{~d} x=\frac{2}{\pi} \int_{0}^{\pi / 2} g_{s}(2 \sin (t)) \mathrm{d} t .
$$

For $s=1$ : equation (31), [BB87, Exercise 1c, p. 16], and Jacobi's imaginary transformations [BB87, Exercises 7a) \& 8b), p. 73] allow us to write

$$
\frac{\pi}{2} g_{1}(x)=(x+1) E\left(\frac{2 \sqrt{x}}{x+1}\right)=\operatorname{Re}\left(2 E(x)-\left(1-x^{2}\right) K(x)\right)
$$

where $K(k)=\int_{0}^{\pi / 2} \mathrm{~d} t / \sqrt{1-k^{2} \sin ^{2}(t)}$ and $E(k)=\int_{0}^{\pi / 2} \sqrt{1-k^{2} \sin ^{2}(t)} \mathrm{d} t$ denote the complete elliptic integrals of the first and second kind. Thus, from (34) and (35),

$$
\begin{aligned}
W_{3}(1)= & \frac{4}{\pi^{2}} \operatorname{Re} \int_{0}^{\pi / 2}\left(2 E(2 \sin (t))-\left(1-4 \sin ^{2}(t)\right) K(2 \sin (t))\right) \mathrm{d} t \\
= & \frac{4}{\pi^{2}} \operatorname{Re} \int_{0}^{\pi / 2} \int_{0}^{\pi / 2} 2 \sqrt{1-4 \sin ^{2}(t) \sin ^{2}(r)} \mathrm{d} t \mathrm{~d} r \\
& -\frac{4}{\pi^{2}} \operatorname{Re} \int_{0}^{\pi / 2} \int_{0}^{\pi / 2} \frac{1-4 \sin ^{2}(t)}{\sqrt{1-4 \sin ^{2}(t) \sin ^{2}(r)}} \mathrm{d} t \mathrm{~d} r
\end{aligned}
$$

Amalgamating the two last integrals and parameterizing, we consider

$$
Q(a):=\frac{4}{\pi^{2}} \int_{0}^{\pi / 2} \int_{0}^{\pi / 2} \frac{1+a^{2} \sin ^{2}(t)-2 a^{2} \sin ^{2}(t) \sin ^{2}(r)}{\sqrt{1-a^{2} \sin ^{2}(t) \sin ^{2}(r)}} \mathrm{d} t \mathrm{~d} r .
$$


We now use the binomial theorem to integrate (36) term-by-term for $|a|<1$ and substitute $\frac{2}{\pi} \int_{0}^{\pi / 2} \sin ^{2 m}(t) \mathrm{d} t=(-1)^{m}\left(\begin{array}{c}-1 / 2 \\ m\end{array}\right)$ throughout. Moreover, $(-1)^{m}\left(\begin{array}{c}-\alpha \\ m\end{array}\right)=(\alpha)_{m} / m$ ! where the later denotes the Pochhammer symbol. Evaluation of the consequent infinite sum produces:

$$
\begin{aligned}
Q(a) & =\sum_{k \geqslant 0}(-1)^{k}\left(\begin{array}{c}
-1 / 2 \\
k
\end{array}\right)\left(a^{2 k}\left(\begin{array}{c}
-1 / 2 \\
k
\end{array}\right)^{2}-a^{2 k+2}\left(\begin{array}{c}
-1 / 2 \\
k
\end{array}\right)\left(\begin{array}{c}
-1 / 2 \\
k+1
\end{array}\right)-2 a^{2 k+2}\left(\begin{array}{c}
-1 / 2 \\
k+1
\end{array}\right)^{2}\right) \\
& =\sum_{k \geqslant 0}(-1)^{k} a^{2 k}\left(\begin{array}{c}
-1 / 2 \\
k
\end{array}\right)^{3} \frac{1}{(1-2 k)^{2}} \\
& ={ }_{3} F_{2}\left(\begin{array}{c}
-\frac{1}{2},-\frac{1}{2}, \frac{1}{2} \\
1,1
\end{array} \mid a^{2}\right) .
\end{aligned}
$$

Analytic continuation to $a=2$ yields the claimed result as per for $s=1$.

For $s=-1$ : we similarly and more easily use (31) and (34) to derive

$$
\begin{aligned}
W_{3}(-1) & =\operatorname{Re} \frac{4}{\pi^{2}} \int_{0}^{\pi / 2} K(2 \sin (t)) \mathrm{d} t \\
& =\operatorname{Re} \frac{4}{\pi^{2}} \int_{0}^{\pi / 2} \int_{0}^{\pi / 2} \frac{1}{\sqrt{1-4 \sin ^{2}(t) \sin ^{2}(r)}} \mathrm{d} t \mathrm{~d} r=V_{3}(-1)
\end{aligned}
$$

Example 5. Theorem 6 allows us to establish the following equivalent expressions for $W_{3}(1)$ :

$$
\begin{aligned}
& W_{3}(1)=\frac{4 \sqrt{3}}{3}\left({ }_{3} F_{2}\left(\begin{array}{c}
-\frac{1}{2},-\frac{1}{2},-\frac{1}{2} \mid \\
1,1
\end{array} \mid \frac{1}{4}\right)-\frac{1}{\pi}\right)+\frac{\sqrt{3}}{24}{ }_{3} F_{2}\left(\begin{array}{c}
\frac{1}{2}, \frac{1}{2}, \frac{1}{2} \\
2,2
\end{array} \mid \frac{1}{4}\right) \\
& =2 \sqrt{3} \frac{K^{2}\left(k_{3}\right)}{\pi^{2}}+\sqrt{3} \frac{1}{K^{2}\left(k_{3}\right)} \\
& =\frac{3}{16} \frac{2^{1 / 3}}{\pi^{4}} \Gamma^{6}\left(\frac{1}{3}\right)+\frac{27}{4} \frac{2^{2 / 3}}{\pi^{4}} \Gamma^{6}\left(\frac{2}{3}\right) .
\end{aligned}
$$

These rely on using Legendre's identity and several Clausen-like product formulae, plus Legendre's evaluation of $K\left(k_{3}\right)$ where $k_{3}:=\frac{\sqrt{3}-1}{2 \sqrt{2}}$ is the third singular value as in [BB87]. More simply but similarly, we have

$$
W_{3}(-1)=2 \sqrt{3} \frac{K^{2}\left(k_{3}\right)}{\pi^{2}}=\frac{3}{16} \frac{2^{1 / 3}}{\pi^{4}} \Gamma^{6}\left(\frac{1}{3}\right)
$$

Using the recurrence presented in Example 1 it follows that similar expressions can be given for $W_{3}$ evaluated at odd integers.

In [BSW10], corresponding hypergeometric closed forms for $W_{4}$ are presented. 
Acknowledgements We are grateful to David Bailey, David Broadhurst and Richard Crandall for helpful suggestions, to Bruno Salvy for reminding us of the existence of [Bar64], Michael Mossinghoff for showing us [Klu1906], and to Peter Donovan for stimulating this research and for much subsequent useful discussion. We also thank the referees for their careful reading and comments. The second author is a fellow of the Research Foundation Flanders (FWO). The work of the third author, as a graduate student, was partially funded by NSF-DMS 0070567.

\section{A Appendix}

\section{A.1 An alternative proof of the series evaluation (9)}

We begin with:

Proposition 3. For complex $s$ with Re $s \geqslant 0$,

$$
W_{n}(s)=n^{s} \sum_{m \geqslant 0}(-1)^{m}\left(\begin{array}{c}
s / 2 \\
m
\end{array}\right)\left(\frac{2}{n}\right)^{2 m} \int_{[0,1]^{n}}\left(\sum_{1 \leqslant i<j \leqslant n} \sin ^{2}\left(\pi\left(x_{j}-x_{i}\right)\right)\right)^{m} \mathrm{~d} \boldsymbol{x} .
$$

Proof. Start with

$$
\begin{aligned}
\left|\sum_{k=1}^{n} e^{2 \pi \mathrm{i} x_{k}}\right|^{2} & =\left(\sum_{k=1}^{n} \cos \left(2 \pi x_{k}\right)\right)^{2}+\left(\sum_{k=1}^{n} \sin \left(2 \pi x_{k}\right)\right)^{2} \\
& =\left(\sum_{i<j}\left(\cos \left(2 \pi x_{i}\right)+\cos \left(2 \pi x_{j}\right)\right)^{2}+\left(\sin \left(2 \pi x_{i}\right)+\sin \left(2 \pi x_{j}\right)\right)^{2}\right)-n(n-2) \\
& =4\left(\sum_{i<j} \cos ^{2}\left(\pi\left(x_{j}-x_{i}\right)\right)\right)-n(n-2) \\
& =n^{2}-4\left(\sum_{i<j} \sin ^{2}\left(\pi\left(x_{j}-x_{i}\right)\right)\right)
\end{aligned}
$$

Therefore, noting that binomial expansion may be applied to the integrand outside a set of $n$-dimensional measure zero,

$$
\begin{aligned}
W_{n}(s) & =\int_{[0,1]^{n}}\left(n^{2}-4\left(\sum_{i<j} \sin ^{2}\left(\pi\left(x_{j}-x_{i}\right)\right)\right)\right)^{s / 2} \mathrm{~d} \boldsymbol{x} \\
& =n^{s} \int_{[0,1]^{n}} \sum_{m \geqslant 0}(-1)^{m}\left(\begin{array}{c}
s / 2 \\
m
\end{array}\right)\left(\frac{2}{n}\right)^{2 m}\left(\sum_{i<j} \sin ^{2}\left(\pi\left(x_{j}-x_{i}\right)\right)\right)^{m} \mathrm{~d} \boldsymbol{x} .
\end{aligned}
$$


Thus the result follows once changing the order of integration and summation is justified. Observe that if $s$ is real then $(-1)^{m}\left(\begin{array}{c}s / 2 \\ m\end{array}\right)$ has a fixed sign for $m>s / 2$ and we can apply monotone convergence. On the other hand, if $s$ is complex then we may use

$$
\lim _{m \rightarrow \infty}\left|\frac{\left(\begin{array}{c}
s / 2 \\
m
\end{array}\right)}{\left(\begin{array}{c}
\operatorname{Re} s / 2 \\
m
\end{array}\right)}\right|=\left|\frac{\Gamma(-\operatorname{Re} s / 2)}{\Gamma(-s / 2)}\right|,
$$

which follows from Stirling's approximation, and apply dominated convergence using the real case for comparison.

We next evaluate the integrals in (37):

Theorem 7. For nonnegative integers $m$,

$$
\int_{[0,1]^{n}}\left(\sum_{i<j} \sin ^{2}\left(\pi\left(x_{j}-x_{i}\right)\right)\right)^{m} \mathrm{~d} \boldsymbol{x}=\left(\frac{n}{2}\right)^{2 m} \sum_{k=0}^{m} \frac{(-1)^{k}}{n^{2 k}}\left(\begin{array}{c}
m \\
k
\end{array}\right) \sum_{a_{1}+\cdots+a_{n}=k}\left(\begin{array}{c}
k \\
a_{1}, \ldots, a_{n}
\end{array}\right)^{2} .
$$

Proof. Denote the left-hand by $I_{n, m}$. As in the proof of Proposition 1 we note that the claim is equivalent to asserting that $2^{2 m} I_{n, m}$ is the constant term of

$$
\left(n^{2}-\left(x_{1}+\cdots+x_{n}\right)\left(1 / x_{1}+\cdots+1 / x_{n}\right)\right)^{m} .
$$

Observe that

$$
\begin{aligned}
\left(n^{2}-\left(x_{1}+\cdots+x_{n}\right)\left(1 / x_{1}+\cdots+1 / x_{n}\right)\right)^{m} & =\left(\sum_{1 \leqslant i<j \leqslant n}\left(2-\frac{x_{i}}{x_{j}}-\frac{x_{j}}{x_{i}}\right)\right)^{m} \\
& =(-1)^{m}\left(\sum_{1 \leqslant i<j \leqslant n} \frac{\left(x_{j}-x_{i}\right)^{2}}{x_{i} x_{j}}\right)^{m} .
\end{aligned}
$$

The result therefore follows from the next proposition.

As before, we denote by 'ct' the operator which extracts from an expression the constant term of its Laurent expansion.

Proposition 4. For any integers $1 \leqslant i_{1} \neq j_{1}, \ldots, i_{m} \neq j_{m} \leqslant n$,

$$
\int_{[0,1]^{n}} \prod_{k=1}^{m} 4 \sin ^{2}\left(\pi\left(x_{j_{k}}-x_{i_{k}}\right)\right) \mathrm{d} \boldsymbol{x}=(-1)^{m} \operatorname{ct} \prod_{k=1}^{m} \frac{\left(x_{j_{k}}-x_{i_{k}}\right)^{2}}{x_{i_{k}} x_{j_{k}}} .
$$


Proof. We prove this by evaluating both sides independently. First, we have

$$
\begin{aligned}
\text { LHS } & :=\int_{[0,1]^{n}} \prod_{k=1}^{m} 4 \sin ^{2}\left(\pi\left(x_{j_{k}}-x_{i_{k}}\right)\right) \mathrm{d} \boldsymbol{x} \\
& =(-1)^{m} \int_{[0,1]^{n}} \prod_{k=1}^{m}\left(e^{\pi \mathrm{i}\left(x_{j_{k}}-x_{i_{k}}\right)}-e^{-\pi \mathrm{i}\left(x_{j_{k}}-x_{i_{k}}\right)}\right)^{2} \mathrm{~d} \boldsymbol{x} \\
& =(-1)^{m} \sum_{\boldsymbol{a}, \boldsymbol{b}}(-1)^{\sum_{k}\left(a_{k}+b_{k}-2\right) / 2} \int_{[0,1]^{n}} e^{\pi \mathrm{i} \sum_{k}\left(a_{k}+b_{k}\right)\left(x_{j_{k}}-x_{i_{k}}\right)} \mathrm{d} \boldsymbol{x} \\
& =\sum_{\boldsymbol{a}, \boldsymbol{b}}(-1)^{\sum_{k}\left(a_{k}+b_{k}\right) / 2} \int_{[0,1]^{n}} \cos \left(\pi \sum_{k}\left(a_{k}+b_{k}\right)\left(x_{j_{k}}-x_{i_{k}}\right)\right) \mathrm{d} \boldsymbol{x}
\end{aligned}
$$

where the last two sums are over all sequences $\boldsymbol{a}, \boldsymbol{b} \in\{ \pm 1\}^{m}$. In the last step the summands corresponding to $(\boldsymbol{a}, \boldsymbol{b})$ and $(-\boldsymbol{a},-\boldsymbol{b})$ have been combined.

Now note that, for $a$ an even integer,

$$
\int_{0}^{1} \cos (\pi(a x+b)) \mathrm{d} x= \begin{cases}\cos (\pi b) & \text { if } a=0, \\ 0 & \text { otherwise. }\end{cases}
$$

Since $a_{k}+b_{k}$ is even, we may apply (38) iteratively to obtain

$$
\int_{[0,1]^{n}} \cos \left(\pi \sum_{k}\left(a_{k}+b_{k}\right)\left(x_{j_{k}}-x_{i_{k}}\right)\right) \mathrm{d} \boldsymbol{x}= \begin{cases}1 & \text { if } \boldsymbol{a}, \boldsymbol{b} \in S, \\ 0 & \text { otherwise },\end{cases}
$$

where $S$ denotes the set of sequences $\boldsymbol{a}, \boldsymbol{b} \in\{ \pm 1\}^{m}$ such that

$$
\sum_{k=1}^{m}\left(a_{k}+b_{k}\right)\left(x_{j_{k}}-x_{i_{k}}\right)=0
$$

as a polynomial in $\boldsymbol{x}$. It follows that

$$
\text { LHS }=\sum_{\boldsymbol{a}, \boldsymbol{b} \in S}(-1)^{\sum_{k}\left(a_{k}+b_{k}\right) / 2}
$$

On the other hand, consider

$$
\text { RHS }:=(-1)^{m} \operatorname{ct} \prod_{k=1}^{m} \frac{\left(x_{j_{k}}-x_{i_{k}}\right)^{2}}{x_{i_{k}} x_{j_{k}}},
$$

and observe that, by a similar argument as above,

$$
(-1)^{m} \prod_{k=1}^{m} \frac{\left(x_{j_{k}}-x_{i_{k}}\right)^{2}}{x_{i_{k}} x_{j_{k}}}=\sum_{\boldsymbol{a}, \boldsymbol{b}} \prod_{k=1}^{m}(-1)^{\left(a_{k}+b_{k}\right) / 2}\left(\frac{x_{j_{k}}}{x_{i_{k}}}\right)^{\left(a_{k}+b_{k}\right) / 2}
$$

where the sum is again over all sequences $\boldsymbol{a}, \boldsymbol{b} \in\{ \pm 1\}^{m}$. From here, it is straight-forward to verify that RHS is equivalent to the expression given for LHS in (39). 
The desired evaluation is now available. On combining Theorem 7 and Proposition 3 we obtain that for $\operatorname{Re} s \geqslant 0$,

$$
W_{n}(s)=n^{s} \sum_{m \geqslant 0}(-1)^{m}\left(\begin{array}{c}
s / 2 \\
m
\end{array}\right) \sum_{k=0}^{m} \frac{(-1)^{k}}{n^{2 k}}\left(\begin{array}{c}
m \\
k
\end{array}\right) \sum_{a_{1}+\cdots+a_{n}=k}\left(\begin{array}{c}
k \\
a_{1}, \ldots, a_{n}
\end{array}\right)^{2} .
$$

This is (9).

Remark 8. We briefly outline the experimental genesis of the evaluation given in Proposition 1. The sequence $2^{2 m} I_{3, m}$ appearing in the proof of Theorem 7 is Sloane's, [Slo09], A093388 where a link to [Ver99] is given. That paper contains the sum

$$
2^{2 m} I_{3, m}=(-1)^{m} \sum_{k=0}^{m}\left(\begin{array}{c}
m \\
k
\end{array}\right)(-8)^{k} \sum_{j=0}^{m-k}\left(\begin{array}{c}
m-k \\
j
\end{array}\right)^{3}
$$

and further mentions that $2^{2 m} I_{3, m}$ is therefore the coefficient of $(x y z)^{m}$ in

$$
(8 x y z-(x+y)(y+z)(z+x))^{m} .
$$

Observe also that $2^{2 m} I_{2, m}$ is the coefficient of $(x y)^{m}$ in

$$
(4 x y-(x+y)(y+x))^{m} .
$$

It was then noted that

$$
8 x y z-(x+y)(y+z)(z+x)=3^{2} x y z-(x+y+z)(x y+y z+z x)
$$

and this line of extrapolation led to the correct conjecture, so that the next case would involve

$$
4^{2} w x y z-(w+x+y+z)(w x y+x y z+y z w+z w x)
$$

which was what we have now proven.

\section{A.2 Numerical evaluations}

A one-dimensional reduction of the integral (1) may be achieved by taking periodicity into account:

$$
W_{n}(s)=\int_{[0,1]^{n-1}}\left|1+\sum_{k=1}^{n-1} e^{2 \pi \mathrm{i} x_{k}}\right|^{s} \mathrm{~d}\left(x_{1}, \ldots, x_{n-1}\right) .
$$

From here, we note that quick and rough estimates are easily obtained using the Monte Carlo method. Moreover, since the integrand function is periodic this seems like an invitation to use lattice sequences - a quasi-Monte Carlo method. E.g., the lattice sequence 
from [CKN06] can be straightforwardly employed to calculate an entire table in one run by keeping a running sum over different values of $n$ and $s$. A standard stochastic error estimator can then be obtained by random shifting.

Generally, however, Broadhurst's representation (28) seems to be the best available for high precision evaluations of $W_{n}(s)$. We close by commenting on the special cases $n=3,4$.

Example 6. The first high precision evaluations of $W_{3}$ were performed by David Bailey who confirmed the initially only conjectured Theorem 6 for $s=2, \ldots, 7$ to 175 digits. This was done on a 256-core LBNL system in roughly 15 minutes by applying tanh-sinh integration to

$$
W_{3}(s)=\int_{0}^{1} \int_{0}^{1}\left(9-4\left(\sin ^{2}(\pi x)+\sin ^{2}(\pi y)+\sin ^{2}(\pi(x-y))\right)\right)^{s / 2} \mathrm{~d} y \mathrm{~d} x,
$$

which is obtained from (40) as in Proposition 3. More practical is the one-dimensional form (34) which can deliver high precision results in minutes on a simple laptop. For integral $s$, Theorem 6 allows extremely high precision evaluations.

Example 7. Assuming that Conjecture 1 holds for $n=2$ (for a proof, see [BSWZ11]), Theorem 6 implies that for nonnegative integers $k$

$$
W_{4}(k) \stackrel{?}{=} \operatorname{Re} \sum_{j \geqslant 0}\left(\begin{array}{c}
s / 2 \\
j
\end{array}\right)^{2}{ }_{3} F_{2}\left(\begin{array}{c}
\frac{1}{2},-\frac{k}{2}+j,-\frac{k}{2}+j \\
1,1
\end{array} \mid 4\right) .
$$

This representation is very suitable for high precision evaluations of $W_{4}$ since, roughly, one correct digit is added by each term of the sum. Formula (30) by Crandall also lends itself quite well for numerical work (by slightly perturbing even $s$ for integer arguments).

\section{References}

[BB10] D. H. Bailey and J. M. Borwein. "Hand-to-hand combat: Experimental mathematics with multi-thousand-digit integrals." Journal of Computational Science, in press, 2010. Available at: http://www.carma.newcastle.edu.au/ jb616/ combat.pdf.

[BBBG08] D. H. Bailey, J. M. Borwein, D. J. Broadhurst, and M. L. Glasser. "Elliptic integral evaluations of Bessel moments and applications." J. Phys. A: Math. Theor., 41, 5203-5231, 2008.

[Bar64] P. Barrucand. "Sur la somme des puissances des coefficients multinomiaux et les puissances successives d'une fonction de Bessel." Comptes rendus hebdomadaires des séances de l'Académie des sciences, 258, 5318-5320, 1964. 
[BBC07] J. M. Borwein, D. Bailey, N. Calkin, R. Girgensohn, R. Luke, and V. Moll. Experimental Mathematics in Action. A.K. Peters, 2007.

[BB87] J. M. Borwein and P. B. Borwein. Pi and the AGM: A Study in Analytic Number Theory and Computational Complexity. Wiley, 1987.

[BS08] J. M. Borwein and B. Salvy. "A proof of a recursion for Bessel moments." Experimental Mathematics, 17, 223-230, 2008. [D-drive Preprint 346.]

[BSW10] J. M. Borwein, A. Straub, and J. Wan. "Three-Step and Four-Step Random Walk Integrals." Experimental Mathematics, in press, 2011. Available at: http: //www. carma.newcastle.edu.au/ jb616/walks2.pdf

[BSWZ11] J. M. Borwein, A. Straub, J. Wan, and W. Zudilin. "Densities of short uniform random walks." Canad. J. Math., in press, 2011. Available at: http://www . carma.newcastle.edu.au/ jb616/densities.pdf

[Bro09] D. Broadhurst. "Bessel moments, random walks and Calabi-Yau equations." Preprint, November 2009.

[CKN06] R. Cools, F. Y. Kuo, and D. Nuyens. "Constructing embedded lattice rules for multivariate integration." SIAM J. Sci. Comput., 28, 2162-2188, 2006.

[Cra09] R. E. Crandall. "Analytic representations for circle-jump moments." Preprint, October 2009.

[Don09] P. Donovan. "The flaw in the JN-25 series of ciphers, II." Cryptologia, in press, 2010.

[Fet63] F. E. Fettis. On a conjecture of Karl Pearson, pages 39-54 in Rider Anniversary Volume, 1963.

[Hug95] B. D. Hughes. "Random Walks and Random Environments," Volume 1. Oxford, 1995.

[Klu1906] J. C. Kluyver. "A local probability problem." Nederl. Acad. Wetensch. Proc., 8, 341-350, 1906.

[Mat01] L. Mattner. "Complex differentiation under the integral." Nieuw Arch. Wisk., IV. Ser. 5/2(1), 32-35, 2001.

[Merz79] E. Merzbacher, J. M. Feagan, and T-H. Wu. "Superposition of the radiation from $N$ independent sources and the problem of random flights." American Journal of Physics. Vol. 45, No. 10, 1977.

[Pea1905] K. Pearson. "The random walk." Nature, 72, 294, 1905. 
[Pea1905b] K. Pearson. "The problem of the random walk." Nature, 72, 342, 1905.

[Pea1906] K. Pearson. A Mathematical Theory of Random Migration, Mathematical Contributions to the Theory of Evolution XV. Drapers, 1906.

[PWZ06] M. Petkovsek, H. Wilf and D. Zeilberger. $A=B$, AK Peters, 3rd printing, 2006.

[Ray1905] Lord Rayleigh. "The problem of the random walk." Nature, 72, 318, 1905.

[RS09] L. B. Richmond and J. Shallit. "Counting abelian squares." The Electronic Journal of Combinatorics, 16, 2009.

[Slo09] N. J. A. Sloane. The On-Line Encyclopedia of Integer Sequences. Published electronically at http://www.research.att.com/sequences, 2009.

[Tit39] E. Titchmarsh. The Theory of Functions. Oxford University Press, 2nd edition, 1939.

[Ver99] H. A. Verrill. "Some congruences related to modular forms." Preprint MPI1999-26, Max-Planck-Institut, 1999.

[Ver04] H. A. Verrill. "Sums of squares of binomial coefficients, with applications to Picard-Fuchs equations." 2004. arXiv:math/0407327v1 [math.CO].

[Wat41] G. N. Watson. A Treatise on the Theory of Bessel Functions, Cambridge University Press, 2nd edition, 1941.

[Wil93] H. Wilf. generatingfunctionology, Academic Press, 2nd edition, 1993. 